\title{
Real Time Acquisition of EMG Signal and Head Movement Recognition
}

\author{
Raisy C D \\ $M$ Tech Student \\ EECE Department \\ ITM University, Gurgaon
}

\author{
Sharda Vashisth \\ Assistant Professor \\ EECE Department \\ ITM University, Gurgaon
}

\author{
Ashok K Salhan \\ Scientist $F$ \\ BID, DIPAS \\ DRDO, Delhi
}

\begin{abstract}
This paper presents the work on a technology for Real time EMG acquisition and recognition of head movement. EMG signal detection and analysis is useful in various clinical and biomedical applications such as generation of control signal for prosthetic tool, but better solutions for detecting noise free EMG signals need to be developed. In this paper a simple and cost effective method for estimating human head movements from neck EMG signals is proposed. In this work, surface EMG signal is acquired from four main neck muscles (right and left sternocleidomastoid muscles and right and left trapezius muscles) using simple computer interface and processed by MATLAB based filter algorithm for online clean display.
\end{abstract}

\section{Keywords}

Electromyography, Real time acquisition, Muscle contractions

\section{INTRODUCTION}

For the assessment and management of patient health, monitoring of physiological and physical signal is essential. Electromyogram is an important health indicator. Electromyography (EMG) signals represent the electrical activity of a muscle during contraction [1]. When nerve sends the signal to initiate muscle contraction a potential is developed across the muscle due to the flow of ions in and out of muscle cells (i.e. movements of electrolytes) and this ionic current is converted into electronic current with $\mathrm{Ag}-\mathrm{AgCl}$ electrodes placed on the surface of the skin of the contracting muscle [2]. Amplitude of EMG signals can be ranged from 0 to $1.5 \mathrm{mV}$ (rms) while its usable energy is between 0 to $500 \mathrm{~Hz}$ frequencies and is dominant in 50 to $150 \mathrm{~Hz}$ range [3].

Various studies $[4,5,6,7,8,9]$ show that electromyography (EMG) activity of sternocleidomastoid (SCM) muscle and head rotation is interdependent. The EMG value of right SCM increased when the head turns to left hand side and for left SCM muscle, the EMG value increased when the head turn to right hand side [8]. Different movements of trapezius muscle can produce voltages with different levels [10]. EMG signals acquired from the trapezius muscles are responsible for flexion and lateral rotation. These signals can be used as the controlling signal for the wheelchair movement. In this work we differentiate three basic head movement by using EMG signal obtained from neck muscles (left and right trapezius and left and right sternocleidomastoid). The identified head movements are flexion, lateral flexion and rotation. Rotation describes the movement of head from side to side, lateral rotation brings ear towards the shoulder and flexion brings the chin to chest as in looking down.

Reminder of this paper is organized as follows: Section 2 presents material and method used for acquiring EMG signal.
Section 3 gives detailed description about the result obtained. And the paper is concluded in Section 4.

\section{MATERIAL AND METHOD}

This section elaborates the material and method required to detect, filter and display human EMG signal. It also explains the requirements of dual channel computer based system to acquire biological signal simultaneously.

\subsection{EMG signal acquisition}

Functional block diagram of Laptop based portable system for real time acquisition of human EMG signal is shown in Figure 1. The system consists of $\mathrm{Ag}-\mathrm{AgCl}$ electrodes, amplifier and filter block and output display unit. Amplifier and filter block is made up of TL-084C operational amplifier and power supply is given to these op-amps by $9 \mathrm{~V}$ batteries [11]. The TL-084C has large common mode rejection ratio (CMRR) and adjustable gain up to 500 [12]. EMG signal picked up by the $\mathrm{Ag}-\mathrm{AgCl}$ electrodes are amplified by buffer amplifiers $\mathrm{A} 1$ and A2 with low gain of 10 . These amplified signals are passed through the unity gain amplifier A3 as shown in Figure 1. A4 is a feedback amplifier with a gain of 50. It helps in dc restoration and gives an overall amplification of 500 to EMG amplifier system. To avoid amplifier saturation an integrator filter A5 is put in feedback. CMRR is increased by the use of amplifier A6 by designing right arm drive circuit [13].

The output of the amplifier and filter block is interfaced directly to the sound port of laptop and EMG signal is viewed through the MATLAB based oscilloscope window after digital filtering. Digital filtering is done by MATLAB programming.

\subsection{Dual channel acquisition system}

Figure 2 shows functional block diagram of computer based dual channel system to simultaneously record two EMG signals. For dual channel acquisition system we need two numbers of amplifiers and filter block, one output display unit and five $\mathrm{Ag}-\mathrm{AgCl}$ electrodes. Analog outputs from EMG amplifier systems are fed to the stereo input jack interfaced to the Laptop through the sound port. Digital processing, storing and visualization of the simultaneously acquired EMG signals is done using MATLAB programming [14]. Figure 3 shows the flow chart of virtual oscilloscope in MATLAB for simultaneous acquisition and filtration of two EMG signals.

In MATLAB program first we create analog input object and then two channels are added to acquire two EMG signals simultaneously at the sound port. After that, set sampling rate as 8000 samples per second and start collecting data. Once the data is obtained, it is segregated to get two separate EMG signals. Pass band range of the FIR filter is set to $1 \mathrm{~Hz}$ to 200 $\mathrm{Hz}$. The two filtered EMG signals are plotted simultaneously. 


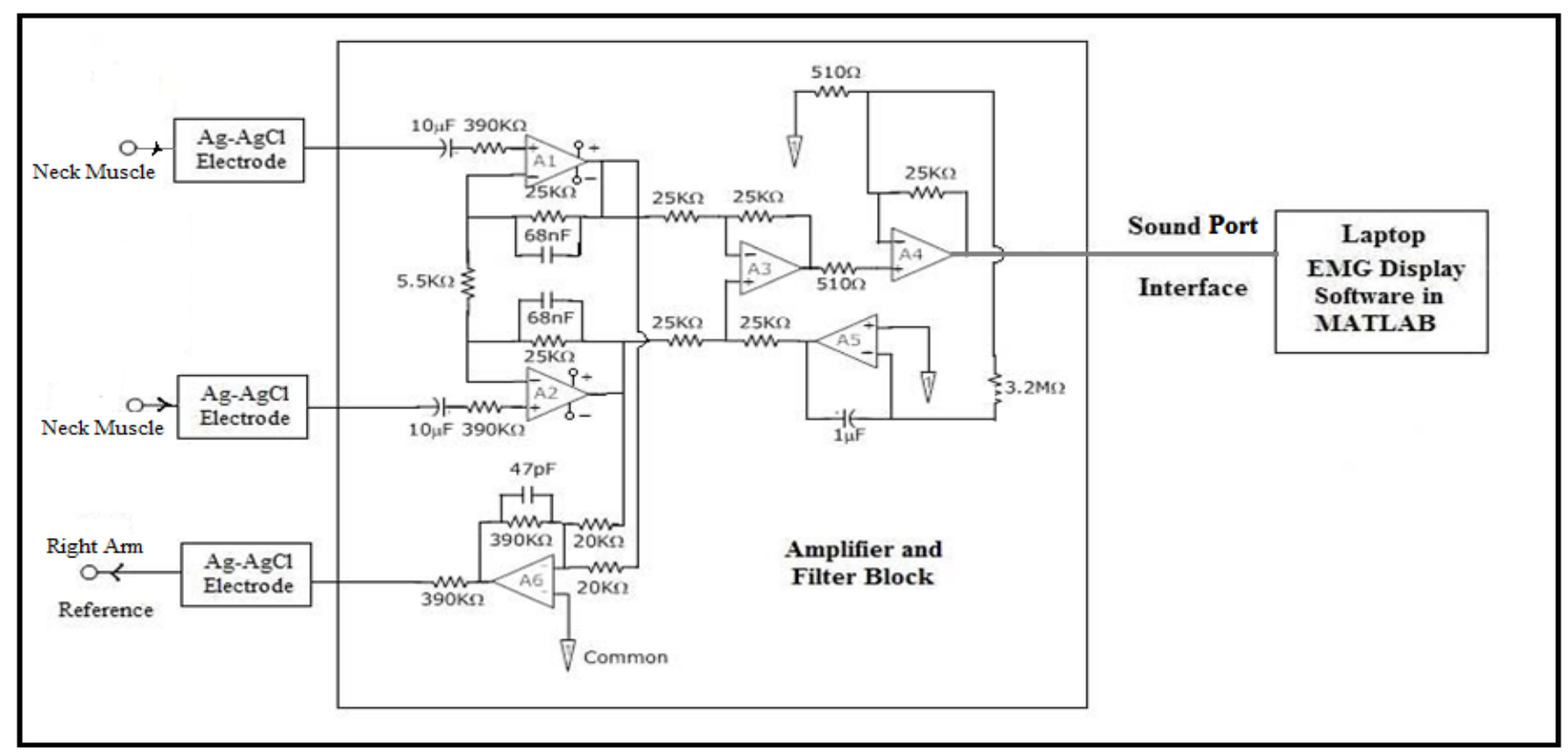

Fig 1: Functional block diagram of real time Acquisition system of human EMG signal

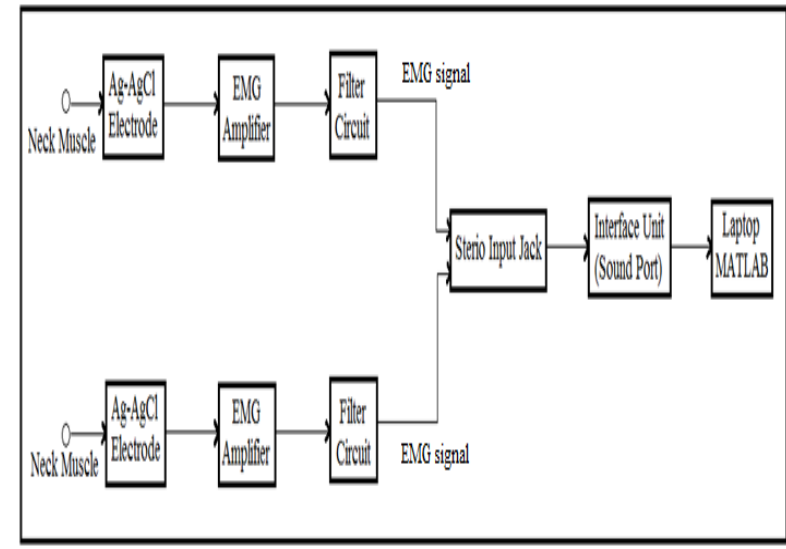

Fig 2: Functional block diagram of computer based dual channel acquisition system

\subsection{Electrode placement}

For taking the EMG signals of trapezius muscle contractions through dual channel acquisition system, two electrodes are placed on the upper part of the left trapezius, two electrodes are placed on the upper part of the right trapezius and one reference electrode is put on the right arm of the human subject. For taking the EMG signals of sternocleidomastoid muscle contractions through dual channel acquisition system, two electrodes are placed on the left sternocleidomastoid, two electrodes are placed on the right sternocleidomastoid and one reference electrode is put on the right arm of the human subject.

\section{RESULTS AND DISCUSSIONS}

Filtered surface EMG signals obtained non-invasively from trapezius and sternocleidomastoid muscles during flexion, lateral rotation and rotation of head is shown in Figure 4 to Figure 8. In Figure 4 to Figure 8, the samples of EMG signals are given on the horizontal axis and corresponding amplitude is given on the vertical axis. As referring to Figure 4 and Figure 5, the amplitude of EMG signals of left and right trapezius are increased when the head is rotated laterally. Figure 4 shows that when the head is laterally rotated towards left the increase in EMG value of left trapezius is more as compared to right trapezius. Figure 5 shows that when the head is laterally rotated towards right the increase in EMG value of right trapezius is more as compared to left trapezius. Figure 6 shows that both the right and left trapezius have similar amplitude during flexion. As referring to Figure 7 and Figure 8, the amplitude of EMG signals of left and right sternocleidomastoid are increased when the head is rotated towards right and left respectively. Figure 7 shows that when the head is rotated towards left the increase in EMG value of right sternocleidomastoid is more as compared to left sternocleidomastoid. Figure 8 shows that when the head is rotated towards right the increase in EMG value of left sternocleidomastoid is more as compared to right sternocleidomastoid.

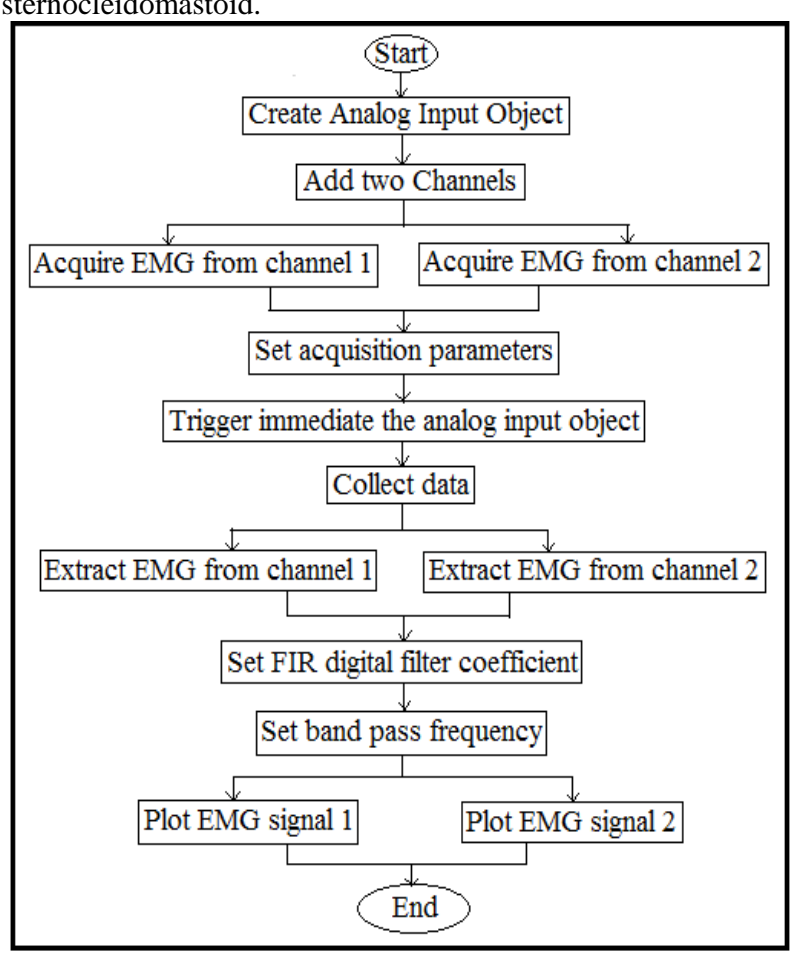

Fig 3: Flow chart for dual channel acquisition of two EMG signals in MATLAB 


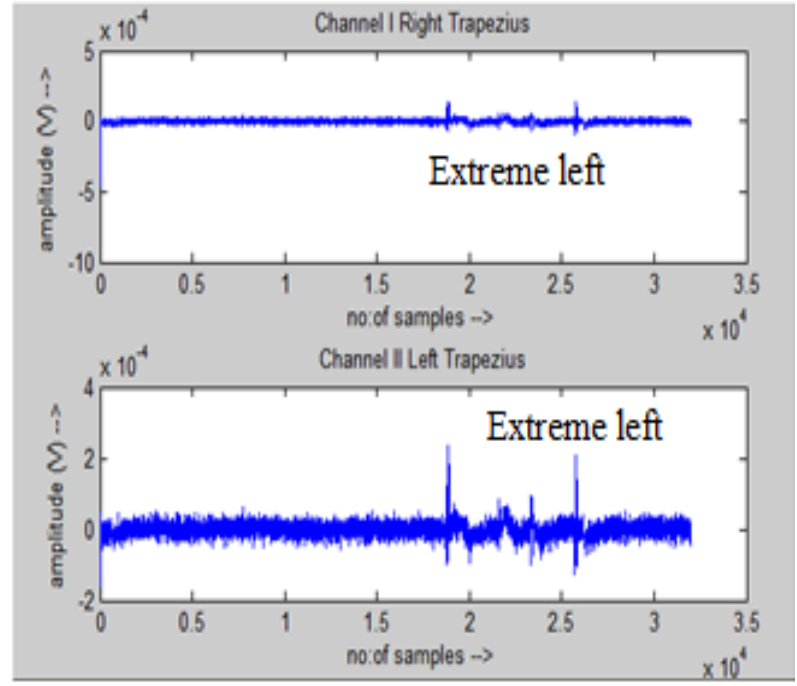

Fig 4: Snap shot of filtered surface EMG acquired from trapezius muscle when head is laterally rotated towards left

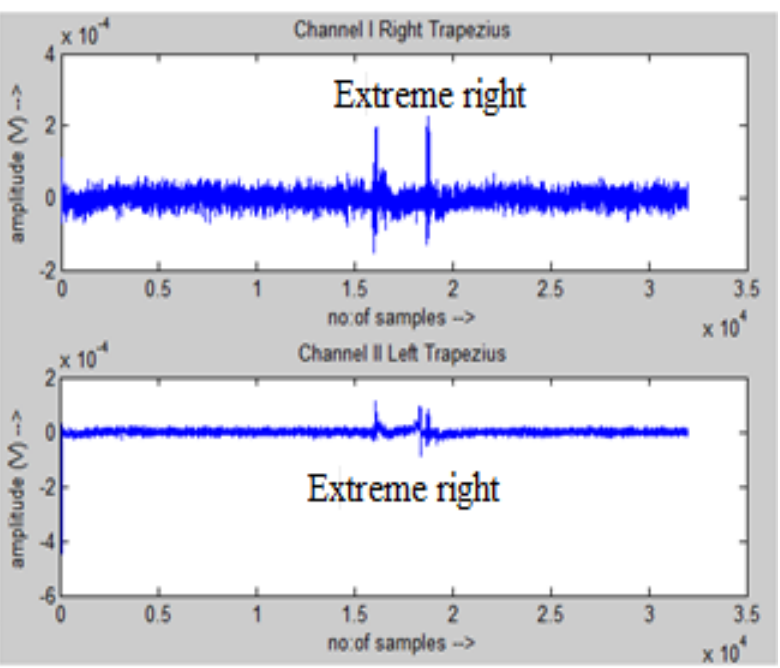

Fig 5: Snap shot of filtered surface EMG acquired from trapezius muscle when head is laterally rotated towards right

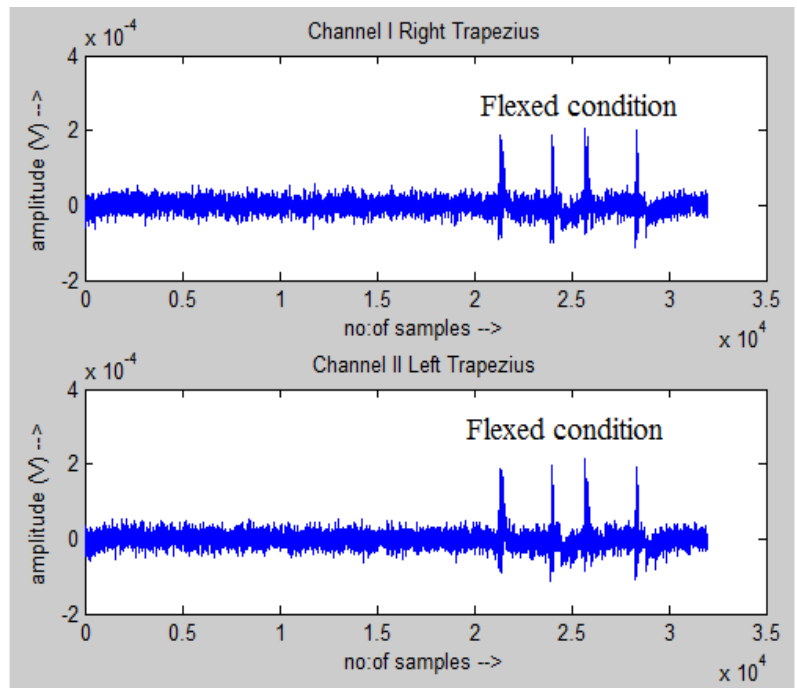

Fig 6: Snap shot of filtered surface EMG acquired from trapezius muscle during flexion

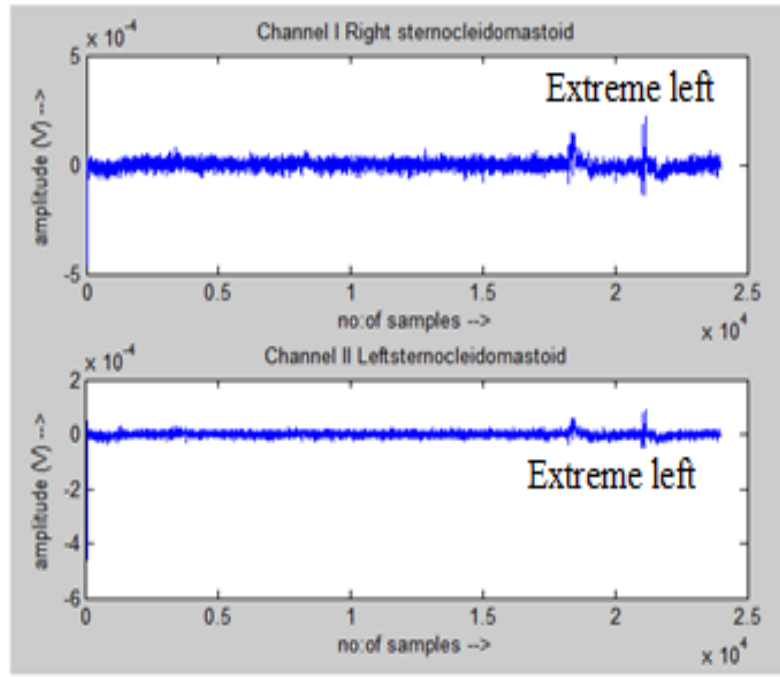

Fig 7: Snap shot of filtered surface EMG acquired from sternocleidomastoid muscle when head is rotated towards left

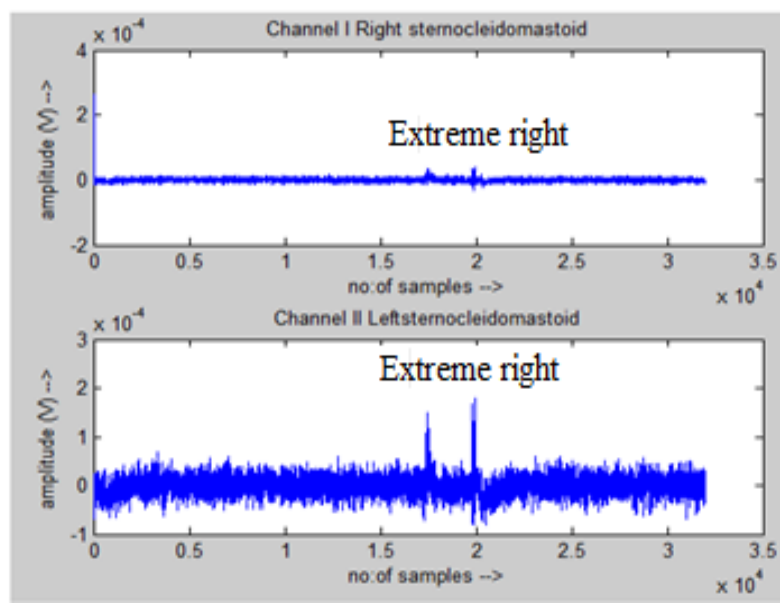

Fig 8: Snap shot of filtered surface EMG acquired from sternocleidomastoid muscle when head is rotated towards right

\section{CONCLUSION}

A simple, cost effective interface system for the online display of EMG signal with the help of laptop is presented in this work. The results show that EMG signal can be detected during contractions of trapezius and sternocleidomastoid muscles in real time and from the acquired EMG signals we can identify the head movements. The obtained signals can be used as control signals for prosthetic tool.

\section{REFERENCES}

[1] Madeleine M. Lowery, Nikolay S. Stoykov, Allen Taflove, Todd A.Kuiken, " A Multiple-layer Finite Element Model of the Surface EMG Signal, "IEEE Transaction on Biomedical Engineering, May 2002, vol 49, no.5, pp446-456.

[2] Taslim Reza, S.M.Ferdous, Md. Nayeemul Hasan, Md. Rokonuzzaman, Kazi Firoz Ahmed, A.Z.M.Shahriar Muttalib, "A Low Cost Surface Electromyogram (sEMG) Signal Guided Automated Wheel Chair for the Disabled", International Journal of Scientific \& Engineering Research, February 2012,vol 3, Issue 2

[3] T. S. Poo, K. Sundaraj, "Design and Development of a Low Cost EMG Signal Acquisition System Using 
Surface EMG Electrode", IEEE Int. conf. Circuits and Systems, Dec 2010, pp. 24-27

[4] J. Ylinen, S. Nuorala, K. Hakkinen, H. Kautiaonen and A. Hakinnen, "Axial neck rotation strength in neutral and prerotated postures", Clinical Biomechanics, 2003, vol. 18 , pp. 467-472.

[5] C.S.M. Bexander, R. Mellor and P.W. Hodges, "Effect of gaze direction on neck muscle activity during cervical rotation”, Exp Brain Res, 2005, vol. 167, pp. 422-432.

[6] D.A. Gabriel, J.Y. Matsumoto, D.H. Davis, B.L. Currier and K.L.An, "Multidirectional neck strength and electromyographic activity for normal controls", Clinical Biomechanics, 2004, vol. 19, pp. 653-658.

[7] I. Moon, K. Kim, J. Ryu and M. Mun, "Face directionbased human-computer interface using image observation and EMG signal for the disabled", Proceeding of the 2003 IEEE International Conference on Robotics \& Automation, 2003, pp 1515-1520.

[8] Y.Lin and W. Huang, "Cervical postures and electromyographic activities of related neck muscles when using a neck support pillow," Journal of Biomechanics, 2008, vol. 40, no. 2, pp. 414.

[9] C.F. Tan, W. Chen and G.W.M. Rauterberg, "The Relationship of Head Rotation Angle and SCM EMG
Value for the Development of AnS2", Proceedings of the World Congress on Engineering, 2010, vol.3, pp 20822085.

[10] V. Reggie Edgerton, S. L. Wolf, Daniel J. Levendowski, Robert I. Jennrich, Roland R. Roy, "EMG Activity in Neck and Back Muscles During Selected Static Postures in Adult males and Females", Physio-therapy Theory and Practice ,1997, pp 179-195.

[11] Dipali Bansal, Munna Khan,Ashok Kumar Salhan,"Wireless Transmission of EMG Signal and Analysis of its Correlation with Simultaneously Acquired Carotid Pulse Wave Using Dual Channel System", Second International Conference on eHealth, Telemedicine, and Social Medicine, IEEE, Feb 2010, pp. 125-129

[12] Dipali Bansal, Munna Khan, Ashok Kumar Salhan,” A computer based wireless system for online acquisition, monitoring and digital processing of ECG waveforms", Computers in Biology and Medicine, 2009, pp $361-367$

[13] J.J. Carr and J.M. Brown. 1998 Introduction to biomedical equipment technology. fourth ed, Prentice Hall, Inc., Englewood Cliffs, NJ.

[14] MATLAB: http://www.mathworks.com. 